\title{
Special Diffractive Optical Elements for Data Encoding
}

\author{
Hamootal Duadi and Zeev Zalevsky
}

School of Engineering, Bar-Ilan University, Ramat-Gan 52900, Israel

\begin{abstract}
With the rising use of optical communication in different commercial as well as military systems, the issue of optical data security becomes eminent. There are two approaches for dealing with this need: using known encoding methods from electronic communication or developing optical encoding methods. In the first approach, the encoding may be done electronically before converting the information to an optical signal. However the ambition for all optical systems has derived setups for optically implementing these methods. In the second approach new optical encoding methods are developed in order to avoid the complexity of converting electrical methods into optical ones. Furthermore, optical encoding methods best utilize the built in properties of optical data transmission. We present a novel approach for designing a phase mask encoded by wavelength and illumination angles. Via simulations we demonstrate a high sensitivity to the decoding parameters.
\end{abstract}

Keywords: Diffractive optical elements, data encoding, optical encryption.

\section{INTRODUCTION}

Due to the ascent in transmission frequencies, there is an ambition to replacing electronic communication systems with optical systems. This change may allow reaching the optical speed limit of several Tera Hertz. When combined optical and electrical systems, the later restricts the throughput speed, hence the goal is to use all optical systems. The topic of encoding information is very important in optical fiber communication and more so in free space communication.

Encoding is needed for network securing confidential data, noise and perturbation resistance and modulation for multiple users in the same transmission medium.

Optical encoding methods either optically implement electrical communication methods, or use new optical methods. Some electrical communication methods that were implemented optically are phase shift keying (PSK) [1, 2] and optical code division multiplexing (OCMD) [3-6]. However the optical setups needed for these methods are still complex or not all-optical. Quantum key distribution draws massive attention in the last decade, since quantum mechanics have capabilities beyond classical computer systems [7, 8]. However, there are no security proofs or standards and many practical limitations exist, such as channel losses, imperfect "qubits" and complex unrealistic detectors [9]. Other methods based on optical properties are random phase decoding [10,11], polarization decoding [12], etc.

In this work we propose an optical technique where the wavelength and illumination angle are used to encode the phase of a diffractive optical element (DOE). Seemingly, the encryption protocol has only two secret variables of wavelength and angle and is therefore comparatively vulner-

*Address correspondence to this author at the School of Engineering, Bar-Ilan University, Ramat-Gan 52900, Israel; Tel: 972-3-531-7394; Fax: 972-3-738-4050; E-mail: hamootal@gmail.com able. However, with the current stat in tunable lasers, it is realistic to create an illumination, in both visible and infra red spectral range, with a precision in wavelength of up to tenths of nanometers. The plurality of options combined with the need to have the correct optical setup at the exact output location makes it difficult for unauthorized decoding.

Let us consider an illumination with a wavelength $\lambda$ and an illumination angle of $\theta$, propagating through air (red ray in Fig. 1). The distance this wave propagates is:

$\Delta l=D / \cos \theta$

where $\mathrm{D}$ is the vertical distance between the input and output planes, which leads to a phase of:

$\phi_{0}=2 \pi D /(\lambda \cos \theta)$

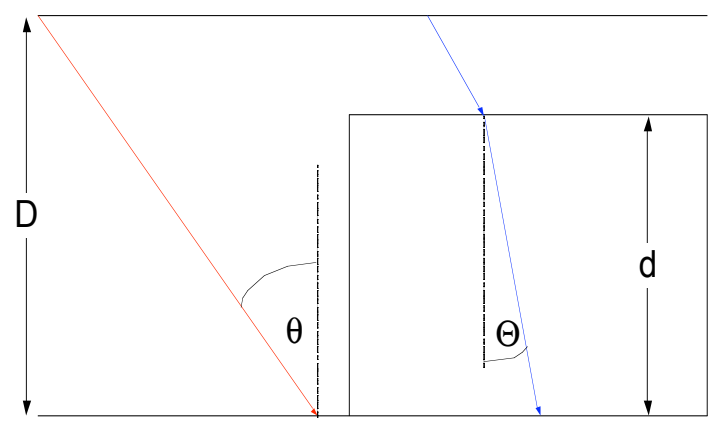

Fig. (1). Illustration of ray path calculations in DOE.

If the same input illumination propagates through a DOE with a refractive index $n$, then a specific pixel with a depth of $d$ (blue ray in Fig. 1) contributed a phase of:

$\phi_{1}=2 \pi(D-d) /(\lambda \cos \theta)+2 \pi n d /(\lambda \cos \Theta)$

where according to Snel's law

$\sin \theta=n \sin \Theta$ 
Hence, the phase addition of each pixel in a DOE is calculated by [13]:

$$
\phi_{1}-\phi_{0}=\phi+2 \pi m=2 \pi \lambda^{-1}\left(n^{2}\left(n^{2}-\sin ^{2} \theta\right)^{-0.5}-(\cos \theta)^{-1}\right) d
$$

where $\mathrm{m}$ is an integer.

Since there is a modulus relation between etching depth and actual phase, by enabling a larger depth one can encode several desired phase distributions in the same element. In this manner different images corresponding to different input illumination wavelengths and angles can be encoded simultaneously, while only an authorized user will receive the correct output image by decoding the spatial information using the proper wavelength and illumination angle.

In this work we will first describe the proposed method. Then, we will plane and test the method via simulation. Finally, we will conclude the findings of this work.

\section{DESCRIPTION OF THE METHOD}

The proposed algorithm is based on the Gerchberg Saxton (GS) algorithm [14]. This algorithm is widely used to retrieve the input phase, from known amplitude distributions of the input and output fields of an optical setup. Later on this algorithm was used for beam shaping applications to compute the required phase element for producing a specific output intensity, given the input illumination. The idea is to choose an initial random phase estimation and compute a new estimation in each new iteration (Fig. 2).

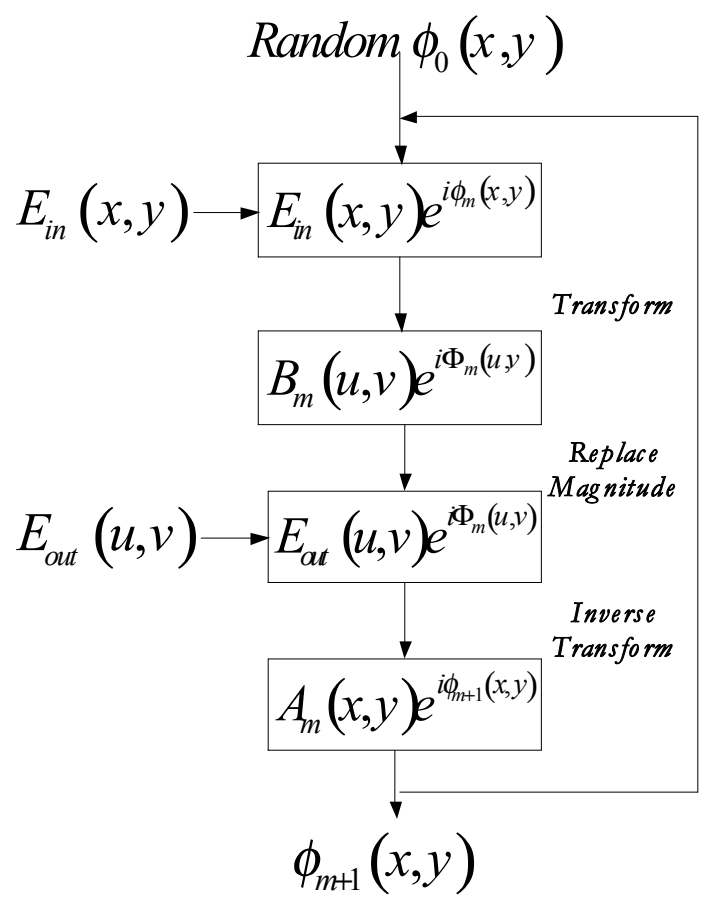

Fig. (2). A scheme of the GS algorithm.

In each iteration the contribution of the current phase estimation and the amplitude of the input illumination are computed, denoted by the input plane expression $\mathrm{E}_{\mathrm{in}}(\mathrm{x}, \mathrm{y}) \exp$ $\left\{i \phi_{\mathrm{m}}(\mathrm{x}, \mathrm{y})\right\}$. Then, the resulting expression after passing through the optical domain is computed by transforming this input plane expression. This is denoted by the output plane expression $\mathrm{B}_{\mathrm{m}}(\mathrm{u}, \mathrm{v}) \exp \left\{\mathrm{i} \Phi_{\mathrm{m}}(\mathrm{u}, \mathrm{v})\right\}$. In order to fulfill the apriori constrain for the output plane intensity, the magnitude of the output plane is replaced by $\mathrm{E}_{\text {out }}(\mathrm{u}, \mathrm{v})$. The output plane expression now becomes $\mathrm{E}_{\text {out }}(\mathrm{u}, \mathrm{v}) \exp \left\{\mathrm{i} \Phi_{\mathrm{m}}(\mathrm{u}, \mathrm{v})\right\}$. The new output plane expression, $\mathrm{E}_{\text {out }}(\mathrm{u}, \mathrm{v}) \exp \left\{\mathrm{i} \Phi_{\mathrm{m}}(\mathrm{u}, \mathrm{v})\right\}$, is then inverse transformed to the input plane, denoted by $\mathrm{A}_{\mathrm{m}}(\mathrm{x}, \mathrm{y}) \exp \left\{\mathrm{i \phi}_{\mathrm{m}+1}(\mathrm{x}, \mathrm{y})\right\}$, and the apriori constrain for the input amplitude $\mathrm{E}_{\mathrm{in}}(\mathrm{x}, \mathrm{y})$ is imposed on the input expression. The phase $\phi_{\mathrm{m}+1}(\mathrm{x}, \mathrm{y})$ is the new phase estimation. This process is repeated until convergence is obtained.

The original GS algorithm was used in a Fourier based optical setup. However, other optical transforms, such as the Fresnel and the fractional Fourier transform, were later on proven to be more efficient in terms of convergence rate [15].

In this work we use the GS algorithm applied in the Fresnel domain to plane several independent phase elements, and later combine them in the same element by planning the optimal etching depth for each pixel. Since the idea of this method is to work with large depths, there is no need to consider all values of the integer $m$ from Eq. (5), but rather a range of high values between $\mathrm{m}_{\min }$ and $\mathrm{M}$. For simplicity, we will demonstrate the design of two different output images, but the proposed method can be implemented for encoding of a plurality of output images. The proposed method is as follows:

1. First, two independent phase elements $\phi_{1}, \phi_{2}$ are planed for different input illumination wavelengths and angles $\lambda_{1}, \theta_{1}$ and $\lambda_{2}, \theta_{2}$ using the GS algorithm applied in the Fresnel domain (explained above).

2. Then, the etching depth of each pixel is computed for different values of $\mathrm{m}=\mathrm{m}_{\min }, \mathrm{m}_{\min }+1, \ldots \mathrm{M}$ by:

$d^{m}\left(x=x_{i}, y=y_{j}\right)=\lambda(2 \pi)^{-1}\left(\phi_{1}\left(x_{i}, y_{j}\right)+2 \pi m\right)\left(n^{2}\left(n^{2}-\sin ^{2} \theta\right)^{-0.5}-(\cos \theta)^{-1}\right)^{-1}$

derived from Eq. (5). As a result, the estimated input phase for the second iteration of the GS algorithm is:

$\tilde{\phi}_{2}^{m}\left(x_{i}, y_{j}\right)=\bmod _{2 \pi}\left[2 \pi \lambda^{-1}\left(n^{2}\left(n^{2}-\sin ^{2} \theta\right)^{-0.5}-(\cos \theta)^{-1}\right) l^{m}\left(x_{i}, y_{j}\right)\right]$

3. The difference between the estimated phase $\tilde{\phi}_{2}^{m}(x, y)$ and the desired one $\phi_{2}(x, y)$ is calculated and denoted by

$\Delta \phi_{2}{ }^{m}(x, y)=\exp \left(i \tilde{\phi}_{2}{ }^{m}(x, y)\right)-\exp \left(i \phi_{2}{ }^{m}(x, y)\right)$

4. For each pixel, the etching depth $d^{m}\left(x_{i}, y_{j}\right)$ corresponding to the minimal value of $\Delta \phi_{2}^{m}\left(x_{i}, y_{j}\right)$ is chosen.

Note that since the depth of a pixel is computed from the first element, there is no added error to the first output image. Furthermore, by choosing the depth of each pixel by the minimum phase difference described in Eq. (8), one minimizes the error of the second output image. This intuitive convergence proof will be demonstrated later on. 

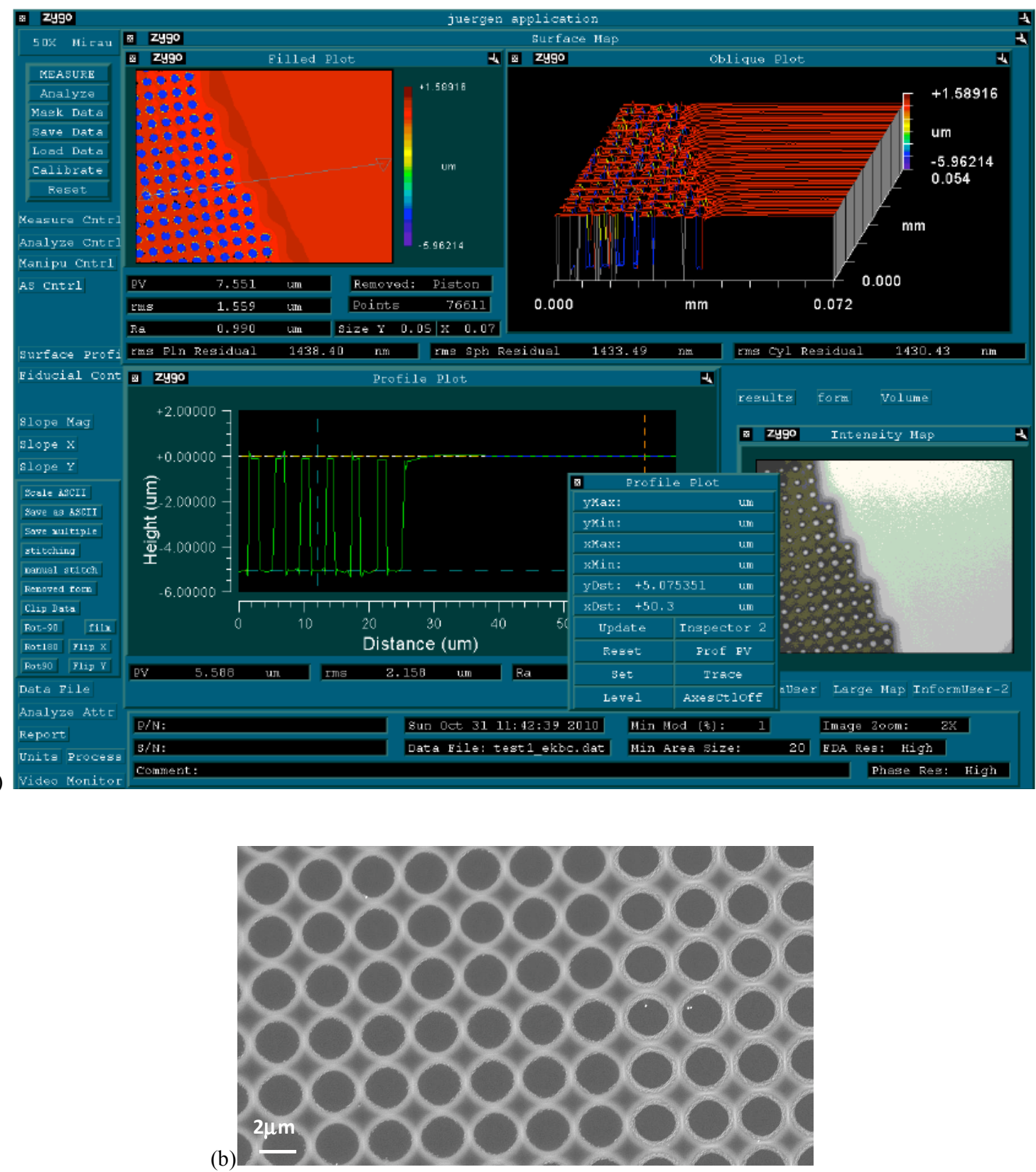

Fig. (3). An example of a fabricated DOE with a high aspect ratio and which was fabricated on silicon wafer using a laser writer machine. (a). Cross section measurements obtained via the Zygo machine. (b) Top view of the element obtained via SEM.

However, there is no guarantee of convergence to all desired images for multiple outputs, but rather for a decrease in the overall error.

The complexity of computation for $p$ output images is the same as computing two output planes. However in the case of multiple outputs a larger range of values is needed for the integer $\mathrm{m}$ in order to enable convergence.

By using this method one may decode pictorial data by a unique optical key, which is the wavelength and illumination angle. The computed depth profile may then be used to fabricate a DOE by using a laser writing machine which can fabricate photonic structures with relatively large aspect ratios (the ratio between the height and the width of the fabricated features as can for example be seen in the fabricated stricture of Fig. 3). Note that the obtainable resolution of the element in Fig. (3) was about $1-2 \mu \mathrm{m}$ in the lateral plane. The fabricated element was generated on a silicon wafer. In Fig. (3a) we present the cross section measurements obtained via the Zygo machine. In Fig. (3b) we show the top view of the element obtained via SEM.

Since the DOE is a passive and static component, this method is most suitable for encoding identification amplitude patterns, such as fingerprint, face detection, signature or retina image.

\section{SIMULATION}

The criteria which we used to evaluate the abilities of the proposed method are the root mean square (RMS) error and 


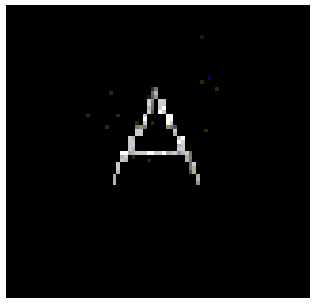

(a)

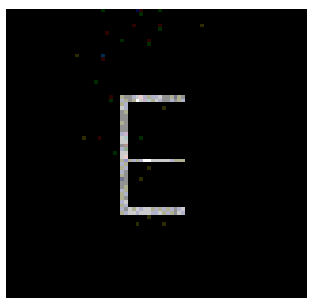

(b)

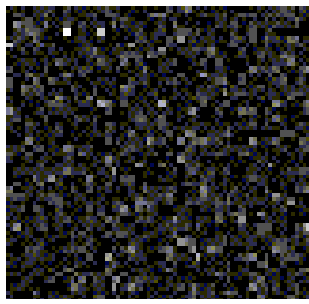

(c)

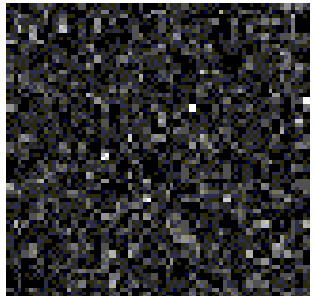

(d)

Fig. (4). Output intensities obtained for the (a) first and (b) second objects when being properly decoded in comparison to the intensities obtained for (c) the correct wavelength and $0.5^{\circ}$ of angular error and (d) the correct angle and $2 \mathrm{~nm}$ of error in the illuminating wavelength.

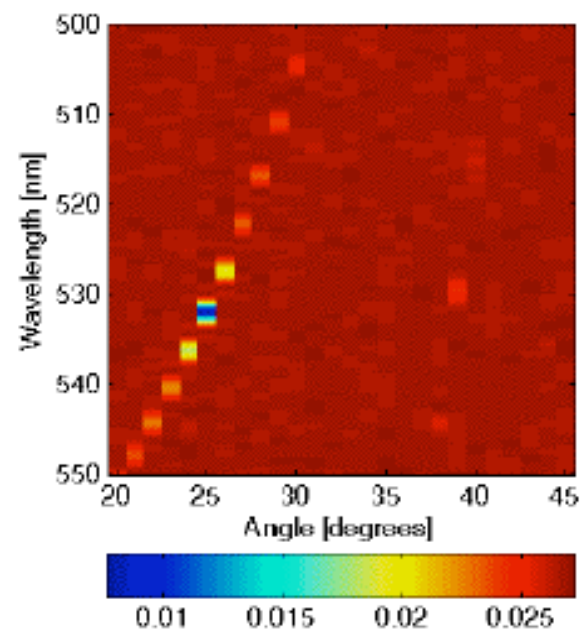

(a)

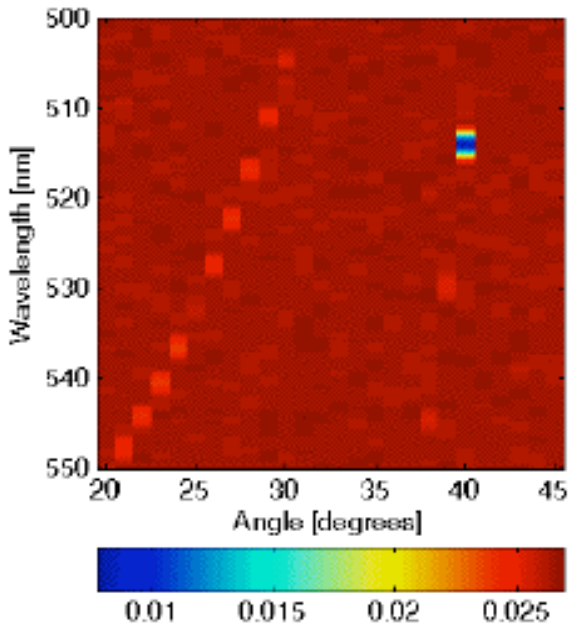

(b)

Fig. (5). RMS error for the first (a) and the second (b) output planes.

the code selectivity. The RMS error, which quantifies the quality of the output, is defined as:

$$
R M S=\sqrt{\sum_{u, v=1}^{N}\left|E_{\text {out }}(u, v)-\tilde{E}_{\text {out }}(u, v)\right|^{2}}
$$

where $E_{\text {out }}(u, v)$ is the desired amplitude, $\tilde{E}_{\text {out }}(u, v)$ is the obtained output image and $u, v$ are the coordinates of the pixels in the output plane.

The code selectivity was quantified by half the range (of wavelengths or angles) in which the RMS error did not increase its minimal value by more than $50 \%$.

In order to demonstrate the abilities of the proposed method, the following simulation was performed: The first desired output distributed, which was the letter "A", was designed for an illumination wavelength of $514 \mathrm{~nm}$ and illumination angle of $40^{\circ}$ (see Fig. 4a). The second desired distribution was the letter "E" and it was designed for an illumination wavelength of $532 \mathrm{~nm}$ and decoding angle of $25^{\circ}$. Two separate phase masks were planed and combined according to the proposed method. The DOE that was planed consists of $50 \times 50$ pixels and each pixel is $1 \mathrm{~mm}$ wide.

The output intensities are presented for the correct illumination conditions (Fig. 4a and $\mathbf{4 b}$ ) as well as for an error of $0.5^{\circ}$ in the angle with the correct wavelength (Fig. $4 \mathrm{c})$ and an error of $2 \mathrm{~nm}$ in the wavelength and a correct angle (Fig. 4d). One may see that these small shifts in the illumination conditions make the data unrecognizable. The RMS error of this element's output with each of the two desired output images was calculated for many possible input angles and wavelengths (Fig. 5). It is clear that the low RMS error regions are extremely localized. Analysis of the last results shows a selectivity of about $2 \mathrm{~nm}$ in wavelength and of about $0.5^{\circ}$ in angle.

\section{CONCLUSIONS}

In this paper we presented a method for planning a diffractive optical element that can encode several desired spatial output distributions, which are highly dependent on the input illumination conditions. We have numerically demonstrated high wavelength $(2 \mathrm{~nm})$ and angular $\left(0.5^{\circ}\right)$ selectivity of the obtained reconstruction. The proposed design technique can be applied for the field of optical encryption.

\section{REFERENCES}

[1] Zalevsky Z, Ben-Yaish S, Guetta E, Beiderman Y, Gannot S Optical realization of Viterbi decoder for communication network. Opt Exp 2007; 15: 3635-49.

[2] Cheolhwan K, Guifang L. Direct-detection optical differential 8level phase-shift keying (OD8PSK) for spectrally efficient transmission. Opt Exp 2004; 12: 3415-21. 
[3] Dang NT, Pham AT, Cheng Z. Performance analysis of spectral amplitude encoding ocdm systems over a linear dispersive optical channel. J Opt Comm Netw 2009; 1: 521-9.

[4] Wang X, Wada N. Spectral phase encoding of ultra-short optical pulse in time domain for OCDMA application. Opt Exp 2007; 15: 7319-26.

[5] Pastor D, Amaya W, García-Olcina R, Sales S. Coherent direct sequence optical code multiple access encoding-decoding efficiency versus wavelength detuning. Opt Lett 2007; 32: 1896-8.

[6] Sun-Jong K, Tae-Jung E, Byeong L, Chang-Soo P. Optical temporal encoding/decoding of short pulses using cascaded longperiod fiber gratings. Opt Exp 2003; 11: 3034-40.

[7] Kurtsiefer C, Zarda P, Halder M, Weinfurter H, Gorman PM, Tapster PR, Rarity JG. Quantum cryptography: A step towards global key distribution. Nature 2002; 419: 450.

[8] Bennett CH, Brassard G, Mermin ND. Quantum cryptography without Bell's theorem. Phys Rev Lett 1992; 68: 557-9.
[9] Brassard G, Lütkenhaus N, Mor T, Sanders B C. Limitations on Practical Quantum Cryptography. Phys Rev Lett 2000; 85: 1330-3.

[10] Cuadrado-Laborde C, Duchowicz R, Torroba R, Sicre EE. Dual random phase encoding: a temporal approach for fiber optic applications. Appl Opt 2008; 47: 1940-6.

[11] Javidi B, Ahouzi E. Optical security system with fourier plane encoding. Appl Opt 1998; 37: 6247-55.

[12] Hübel H, Vanner MR, Lederer T, et al. High-fidelity transmission of polarization encoded qubits from an entangled source over 100 $\mathrm{km}$ of fiber. Opt $\operatorname{Exp} 2007$; 15: 7853-62.

[13] Born M, Wolf E. Principles of optics. Cambridge: Cambridge University Press 2001.

[14] Gerchberg RW, Saxton WO. A practical algorithm for the determination of phase from image and diffraction plane pictures. Optik 1972; 35: 237-46.

[15] Zalevsky Z, Mendlovic D, Dorsch RG. The Gerchberg-Saxton algorithm applied in the fractional Fourier or Fresnel domains. Opt Lett 1996; 21: 842-4.

(C) Duadi and Zalevsky; Licensee Bentham Open.

This is an open access article licensed under the terms of the Creative Commons Attribution Non-Commercial License (http://creativecommons.org/licenses/by$\mathrm{nc} / 3.0 /$ ), which permits unrestricted, non-commercial use, distribution and reproduction in any medium, provided the work is properly cited. 